\title{
SPECIAL TOPICS
}

\begin{abstract}
Editor's Note Although Severe Acute Respiratory Syndrome (SARS) situation has been brought under effective control around the world, this contagious disease that caused awful terror and harm still remains a mystery. Where does the pathogen come? How are people infected with the disease? How does the disease spread among people? What is the pathogenesis of SARS? There are many things that need to be understood. With thorough and broad investigations on SARS virus, the scientists in China are gaining more and more scientific achievements. Chinese Science Bulletin, an academic journal, has an obligation to timely communicate these achievements to researchers in China and abroad. From this issue, we will collectively publish the recently accepted contributions on SARS virus research in a new column called "Special Topics" and hope it may help promote the relevant researches. The latest SARS-related results in this issue come from four articles: Shi Rong et al. designed and adopted 60 mer oligonucleotide microarray to detect SARS virus, providing a new method for quick and early SARS diagnosis; Gao Lei et al. and Qi Zhen et al. studied the origin and evolution of SARS coronavirus by different methods and drew some enlightening conclusions; Jia Guangle et al. introduced the three zoonoses caused by fruit bats, which may give an inspiration to SARS epidemiological investigation.
\end{abstract}

Chinese Science Bulletin 2003 Vol. 48 No. $121165-1169$

\section{Design and application of 60mer oligonucleotide microarray in SARS coronavirus detection}

\author{
SHI Rong ${ }^{1}$, MA Wenli ${ }^{1}$, WU Qinghua ${ }^{1}$, ZHANG Bao ${ }^{1}$, \\ SONG Yanbin ${ }^{1}$, GUO Qiuye ${ }^{1}, X^{2}$ XO Weiwei ${ }^{1}$, \\ WANG Yan $^{1} \&$ ZHENG Wenling ${ }^{2}$ \\ 1. Institute of Molecular Biology, First Military Medical University, \\ Guangzhou 510515, China; \\ 2. Department of Medical Research, Guangzhou Liu Hua Qiao Hospital, \\ Guangzhou 510010, China \\ Correspondence should be addressed to Ma Wenli (e-mail: wenli@) \\ fimmu.edu.cn)
}

\begin{abstract}
The 60mer oligonucleotide microarray was designed and applied to detecting of SARS (severe acute respiratory syndrome) coronavirus. Thirty $60 \mathrm{mer}$ specific oligos were designed to cover the whole genome of the first submitted coronavirus strain, according to the sequence of TOR2 (GENEBANK Accession: AY274119). These primers were synthesized and printed into a microarray with $12 \times 12$ spots. RNAs were extracted from the throat swab and gargling fluid of SARS patients and reverse-transcripted into the double strand cDNAs. The cDNAs were prepared as restricted cDNA fragments by the restriction display (RD) technique and labeled by PCR with the Cy5-universal primer. The labeled samples were then applied to the oligo microarray for hybridization. The diagnostic capability of the microarray was evaluated after the washing and scanning steps. The scanning result showed that samples of SARS patients were hybridized with multiple SARS probes on the microarray, and there is no signal on the negative and blank controls. These results indicate that the genome of SARS coronavirus can be detected in parallel by the $60 \mathrm{mer}$ oligonucleotide microarray, which can improve the positive ratio of the diagnosis. The oligo microarray can also be used for monitoring the behavior of the virus genes in different stages of the disease status.
\end{abstract}

Keywords: SARS coronavirus, oligonucleotide microarray, RD technique, fluorescent labeling, molecular hybridization.

DOI: 10.1360/03wc0216

In April 2003, a novel coronavirus ${ }^{[1,2]}$ which was associated with cases of Severe Acute Respiratory Syndrome (SARS) was first isolated and sequenced in Canada. The genome of SARS coronavirus (SARS-CoV) is $29727^{[3]}$ nucleotides in length and has 11 known open reading frames (ORFs). Although the genome organization of this virus is similar to that of other coronaviruses, phylogenetic analyses and sequence alignment show that SARS-CoV is not closely related to any of the previously characterized coronaviruses ${ }^{[3,4]}$. It shows more homology with the bovine coronaviruses and murine hepatitis virus than that of the human strain of the coronaviruses, which suggested that it was not originated from a host of the Homo sapiens ${ }^{[5]}$.

The epidemic situation of SARS is now at a platform stage since it has broken out in several provinces of China. New cases every day were more than 50 and total cases reported reached 4948 up to May 12, 2003. As the incubation period for the disease can last 2 or 3 weeks, early and rapid diagnostic approaches are very important for quarantine of the source of infection and protection of the healthy population. Currently, laboratory examinations were mainly immunological means such as ELISA, colloidal gold indicator paper, or molecular biological methods such as nested RT-PCR ${ }^{[6]}$ or real-time PCR. In this research we designed and manufactured an oligonucleotide microarray, which consisted of thirty 60 mer specific oligonucleotides according to the sequence of Strain $\mathrm{TRO} 2$ to represent the genome of SARS coronavirus. Our studies showed that SARS oligo microarrays yielded better diagnostic results

\section{Materials and methods}

( i ) Source of samples. Four throat swab and gargling fluid of SARS patients were obtained from 302 Hospital in Beijing, the other 3 samples from Liu Hua 


\section{REPORTS}

Qiao Hospital in Guangzhou and 6 samples of normal person were used as negative controls. The samples were centrifuged and the supernatant was stored at $-80^{\circ} \mathrm{C}$. Virus extraction solution was self-prepared, reverse transcriptase SuperScript II was from Invitrogen Co. Random Primer pd(N)6, RNase Inhibitor, E. coli DNA Pol I, E. coli RNase $\mathrm{H}$, E. coli DNA Ligase, restriction enzyme Sau $3 \mathrm{AI}$, Taq DNA polymerase and T4 DNA ligase were from TaKaRa Bio Inc. Universal primer (5' GTTTGGCTGGTGTGGATC $3^{\prime}$ ), adaptor (primer SIP: 5'pGATC ${ }^{\mathrm{m}} \mathrm{CACACCAG-}$ CCAAACCCA3'; SIR: 5 'GGTTTGGCTGGTGTG3 ') were synthesized by BIOASIA Co. Cy 5 labeled universal primer ( $5^{\prime} \mathrm{Cy} 5$-UTTGGCTGGTGTGGATC $3^{\prime}$ ) was synthesized by Trilink Biotechnologies, Inc. Other reagents were: DEPC and Poly-L-Lysine (Sigma), silanlized slides (DAKO), DMSO and Formamide (Amresco), succinic anhydride (Aldrich), 1-methyl-2-pyrrolidinone (Aldrich), sodium borate (Merk-Schuchardt). The oligo probes were synthesized by our institute using an ABI 3900 DNA Synthesizer.

(ii ) Extration of virus RNAs. The RNAs of SARS-CoV were extracted by a Phenol/Freeze protocol, the samples were treated with water saturated phenol at $65^{\circ} \mathrm{C}$ for $10 \mathrm{~min}$ and put into ice bath immediately until phenol crystals appeared, the RNAs were then phenol /chloroform extracted, ethanol-precipitated, and resuspended in $10 \mu \mathrm{L}$ DEPC treated water.

(iii) Reverse transcripted into the ds cDNAs. $\quad 5 \mu \mathrm{L}$ RNAs were mixed with $5 \mu \mathrm{L}$ dNTPs $(10 \mathrm{mmol} / \mathrm{L}), 10 \mu \mathrm{L}$ $5 \times$ first strand buffer, $2.5 \mu \mathrm{L}$ RNase inhibitor, $5 \mu \mathrm{L}$ random primer $\operatorname{pd}(\mathrm{N}) 6,2.5 \mu \mathrm{L}$ SuperScript II , DEPC treated water up to a total volume of $50 \mu \mathrm{L}$. The mixture was kept in water bath at $42^{\circ} \mathrm{C}$ for $50 \mathrm{~min}$, then added with $25 \mu \mathrm{L}$ $10 \times$ second strand buffer, $4 \mu \mathrm{L}$ E. coli DNA Pol I, $1 \mu \mathrm{L}$ $E$. coli $\mathrm{RNase} \mathrm{H}, 1.5 \mu \mathrm{L} E$. coli DNA ligase into the first strand mixture and $\mathrm{ddH}_{2} \mathrm{O}$ up to a total volume of $250 \mu \mathrm{L}$, incubated in water bath at $12^{\circ} \mathrm{C}$ for $1 \mathrm{~h}$, then $22^{\circ} \mathrm{C}$ for $1 \mathrm{~h}$, phenol/chloroform $(25: 24)$ extracted, ethanol-precipitated, the resulting ds cDNA pellet was dissolved in $10 \mu \mathrm{L}$ of $\mathrm{ddH}_{2} \mathrm{O}$.

(iv) Digestion of the cDNAs by restriction enzyme.

All the cDNAs were taken and $1.6 \mu \mathrm{L}$ Sau3A I , 2.4 $\mu \mathrm{L} 10 \times \mathrm{H}$ buffer and $10 \mu \mathrm{L} \mathrm{ddH}_{2} \mathrm{O}$ were added to a total volume of $24 \mu \mathrm{L}$ at $37^{\circ} \mathrm{C}$ for $4 \mathrm{~h}$. The enzyme was inactivated in $100^{\circ} \mathrm{C}$ for $2 \mathrm{~min}$. All the digested fragments had a cohesive end of GATC on both sides.

( v ) Add adaptors to the digested fragments. The two primers SIP $(500 \mu \mathrm{g} / \mu \mathrm{L})$ and SIR $(600 \mu \mathrm{g} / \mu \mathrm{L})$ were denatured at $90^{\circ} \mathrm{C}$ for $5 \mathrm{~min}$ and cooled down to $20^{\circ} \mathrm{C}$ gradually in $30 \mathrm{~min}$ for annealing so that the doublestranded universal adaptors were formed. $5 \mu \mathrm{L}$ digested ds
cDNAs , $3 \mu \mathrm{L}$ adaptors, $2 \mu \mathrm{L}$ T4 DNA ligase, $3 \mu \mathrm{L}$ of 10 $\times \mathrm{T} 4$ DNA ligase buffer and $17 \mu \mathrm{L} \mathrm{ddH}_{2} \mathrm{O}$ were added to the Eppendorf tube and the mixture was kept at $16^{\circ} \mathrm{C}$ for $2 \mathrm{~h}$. The production was purified by Pharmacia S- 400 centrifuge tube to remove the adaptors and short fragments. The eluted solution was collected as a template for fluorescent labeling.

(vi) Fluorescent labeling of the adaptor-ligated ds cDNAs. Cy5 labeled universal primer was designed according to the sequences of the adaptor and restriction site of Sau 3 A I . The labeling reaction included $25 \mu \mathrm{L} 2 \times$ PCR buffer $(100 \mathrm{mmol} / \mathrm{L} \mathrm{KCL}, 20 \mathrm{mmol} / \mathrm{L}$ Tris-HCL PH $\left.8.3,3 \mathrm{mmol} / \mathrm{L} \mathrm{MgCl}_{2}\right), 1 \mu \mathrm{L}$ adaptor-ligated ds cDNA fragments, $3 \mu \mathrm{L}$ Cy5 labeled universal primer, $20 \mu \mathrm{L}$ $\mathrm{ddH}_{2} \mathrm{O}, 1 \mu \mathrm{L}$ Taq DNA polymerase. And the PCR amplifications were performed in an ABI PCR System 9700 with an initial denaturing temperature of $95^{\circ} \mathrm{C}$ for $5 \mathrm{~min}$, followed by 25 cycles of $30 \mathrm{~s}$ at $95^{\circ} \mathrm{C}, 30 \mathrm{~s}$ at $60^{\circ} \mathrm{C}, 1 \mathrm{~min}$ at $72^{\circ} \mathrm{C}$, and a final extension of $5 \mathrm{~min}$ at $72^{\circ} \mathrm{C}^{[7]}$.

(vii) Design of the oligos and microarray. Thirty $60 \mathrm{mer}$ oligos were designed according to the following principles: i) $T_{\mathrm{m}}$ values of all oligos are within (78 \pm 5) ${ }^{\circ} \mathrm{C}$. ii ) The contiguous single nucleotide cannot tract longer than 6 bases. iii) The hairpin structure length cannot be longer than 6 . iv) Each oligo has less than $40 \%$ identity to all other sequences in BLAST comparation. v ) Each oligo cannot have more than 20 contiguous bases similar to any other sequences in BLAST comparison. A $12 \times 12$-spot microarray was designed by all these $60 \mathrm{mer}$ oligos with 3 negative and 3 blank controls. The composition and characteristics of the oligos are shown in Table 1. The scheme of the microarray is shown in Fig. 1.

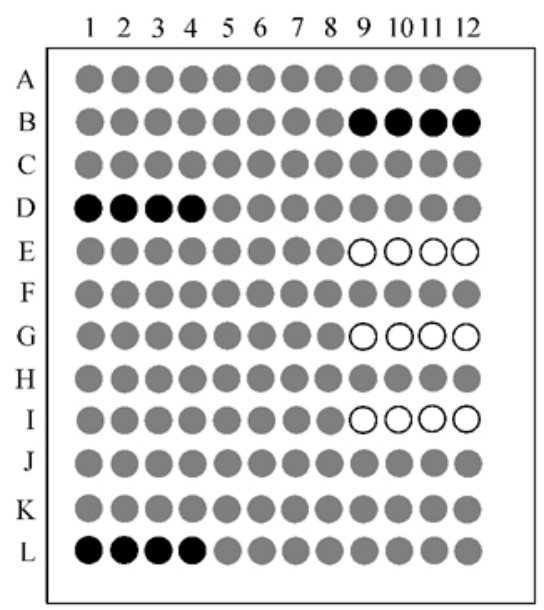

Fig. 1. Scheme of the microarray. OSARS probes; negative controls; Oempty controls. 


\section{SPECIAL TOPICS}




\section{REPORTS}

(vii) Preparation of the 60mer oligonucleotide microarray. The DAKO silanlized slides were coated by Poly-L-Lysine according to the protocol of Brown's lab of Stanford University and stored at room temperature until ready for use. The oligos were synthesized by an ABI 3900 DNA synthesizer and dissolved in 50\% DMSO to reach a final concentration of $1 \mu \mathrm{g} / \mu \mathrm{L}, \mathrm{HBV} 70$ mer olgios were used as negative controls and $\mathbf{5 0} \%$ DMSO were used as blank controls. The microarrays were printed by a Cartesian Pixsys 5500 robot, re-hybridized above the $3 \times$ SSC solution faced down and placed in a baker of $140^{\circ} \mathrm{C}$ for snap dry. BIO-RAD UV cross-linker was used to immobilize the probes onto the slides with $65 \mathrm{~mJ}$. The slides were treated with the blocking solution $(335 \mathrm{~mL}$ 1-methyl2-pyrrolidinone, $6 \mathrm{~g}$ succinic anhydride and $15 \mathrm{~mL} 1$ $\mathrm{mol} / \mathrm{L} \mathrm{NaBorate}, \mathrm{pH} 8$ ) and stored for later use

(viii) Hybridization and scanning. $5 \mu \mathrm{L}$ Cy5 labeled samples were taken to mix with an equal volume of $2 \times$ hybridization buffer $(50 \%$ Formamide, $10 \times \mathrm{SSC}, 0.2 \%$ SDS). The mixture was denatured at $95^{\circ} \mathrm{C}$ for $5 \mathrm{~min}$ and spun to cool down for $2 \mathrm{~min}$ at the maximum speed. $3 \mu \mathrm{L}$ of the samples were pipetted onto the microarray, a silicone pre-treated cover slip was placed on the top of each array and $10 \mu \mathrm{L} \mathrm{ddH_{2 }}$ O were added to the two holes in the Corning CMT hybridization chamber to maintain the humidity. After that, the hybridization chamber was sealed and submerged in a $42^{\circ} \mathrm{C}$ water-bath for $2 \mathrm{~h}$. The slides were washed in $2 \times \mathrm{SSC} / 0.1 \% \mathrm{SDS}, 0.1 \times \mathrm{SSC} / 0.1 \% \mathrm{SDS}$, $0.1 \times \mathrm{SSC}$ by turns, rinsed by $\mathrm{ddH}_{2} \mathrm{O}$ and dehydrated by anhydrous alcohol, then scanned by a Packard Scanarray
Lite scanner.

\section{Result}

The hybridization results of 7 SARS clinical samples and 1 negative normal person control under a scanning condition of $90 \%$ laser power and $72 \%$ PMT are shown in Fig. 2, among which (a), (b), (c), (d) are four SARS samples from 302 Hospital in Beijing, (e), (f), (g) are 3 samples from Liu Hua Qiao Hospital in Guangzhou. Several probe sites showed positive in each microarray. oligo01, oligo03, oligo05, oligo07, oligo10, oligo11, oligo 12, oligo 14, oligo 16, oligo17, oligo 18, oligo19, oligo20, oligo26, oligo27, oligo28, oligo29 and oligo30 were the sites in common for most samples. No signal was shown in the negative and blank control probe sites. While on probe sites of oligo12, oligol1 and oligo 28 , there were signals when we applied the 6 samples of normal person to the microarray.

\section{Discussion}

Researchers are now focusing on the early, rapid, sensitive etiological diagnosis, effective prevention and satisfactory treatment of SARS after coronavirus had been determined to be the pathogen of the disease. Immunology methods are often effective in later stages of the diseases, because they all depended on the emerging of antibodies in the serum. Methods based on PCR have a merit of convenience and easy to use, but they are often designed to amplify only one fragment of the SARS genome, which means that the result would be affected if the samples were not well preserved and treated. DNA microarray for diagnosis is based on the high throughput detection of
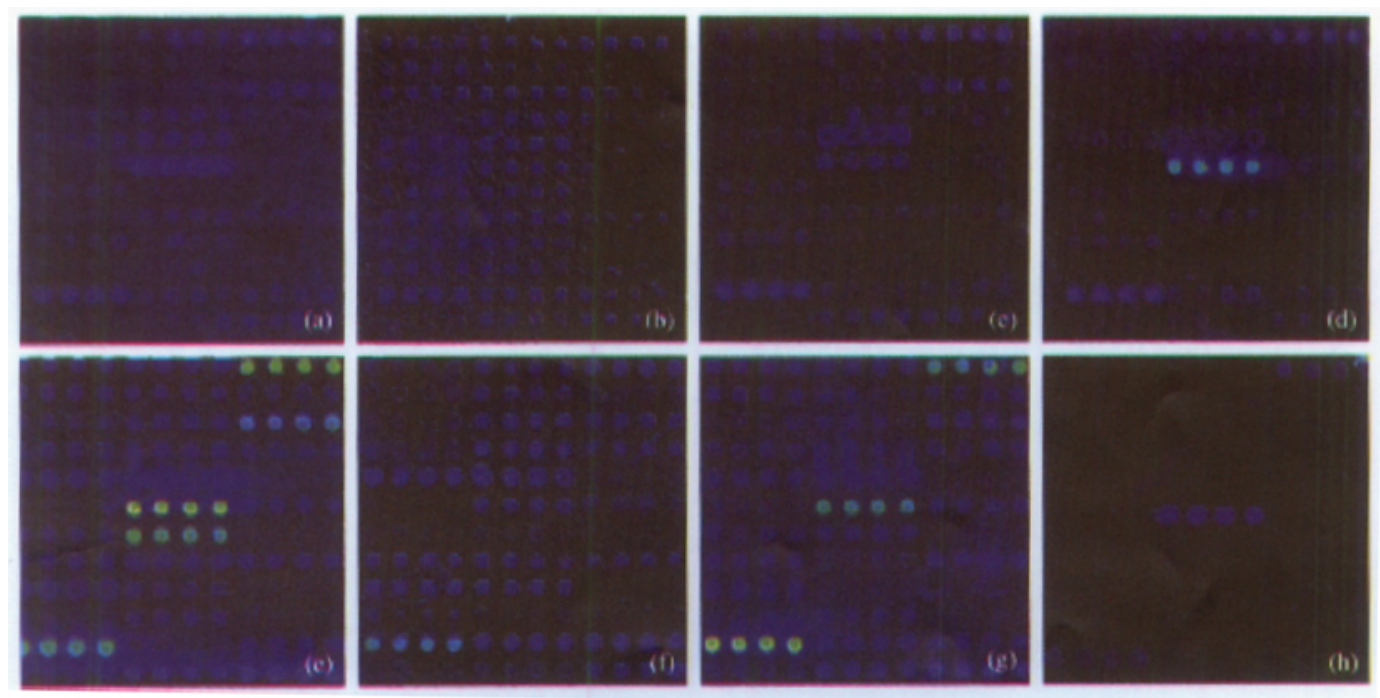

Fig. 2. Results of microarray hybridization. (a) - (g) Hybridization results of the samples from SARS patients; (h) hybridization results of the sample from one normal person. 


\section{SPECIAL TOPICS}

multiple targeting locus. It can help to increase the diagnostic ratio while monitoring the activities of virus genes during the progress of the disease at the same time.

We designed 30 specific $60 \mathrm{mer}$ oligonucleotides to represent the whole genome of SARS coronavirus in this research, and printed them into an oligo microarray, which was applied to hybridizing with the samples of SARS patients treated and labeled by RD-PCR. We can judge from the results of the 7 samples hybridized with the probes on the microarray and there was no signal on the negative and blank probe sites. Although there were some differences among each sample, some common features were shown in the hybridization picture. For example, four samples including (a), (b), (e), (g) were hybridized with the specific probes on site $\mathrm{J5}-\mathrm{J} 8$, which only belonged to SARS coronavirus and could provide very important clues for final diagnosis. However, the differences between different samples might mainly result from the methods for sample storage and treatment. As an RNA virus, the nucleic acids of SARS coronavirus are very easy to de grade, and the ingredients in sputum are very complicated and may include many enzymes and bacteria. If we stored or treated the samples improperly, some of the targeting sequence would be lost unexpectedly. Furthermore, although the RD technique is a method to amplify the whole genome in theory, there is still some possibility of the fragments, which could not be ligated with the adaptors, especially when there was degradation in the samples. This would cause preferential amplification, with some fragments amplified well while other fragments inhibited. These effects were very significant when we observed Fig. 2(c) and Fig. 2(d). That is the reason why the extraction of the virus RNAs from fresh samples and immediately stored in low temperature are very important for improving the accuracy of diagnosis. On the other hand, we had observed some signals in the sites of oligo 12, oligol1 and oligo28, which were hybridized with the sample of normal person, this might be due to the defect of stringency. When we checked the oligos, complementation was found between the two oligos with the flourscent labeled primers of 10 and 11 bases correspondingly. As for probe oligo 11, although the condition of which satisfied the principles we used to design the oligos, a discontinuous 22-base homology presented between the oligo and human BAC clone RP11-333O1, and these led to the non-specific hybridization. That was why all these three probes should be eliminated when the array was used for diagnosis.

Although oligo fragments are short and hard to immobilize onto the glass substances, oligonucleotide microarray still has a character of high specificity when compared with the genome DNA microarray, and it is superior in improving the accuracy in diagnosis of the pathogens. Most of the 30 oligonucleotides we designed were sequences specifically belonging to SARS coronavirus. In order to detect the virus with parallel and specificity, part of the oligos are the common sequences of several coronaviruses and one oligo is a sequence belonging to SARS-CoV absolute specifically which would be helpful in identification of the virus. Furthermore, we can design oligos directly from the known sequence information without the necessity of isolating the pathogen and acquiring of the whole genome DNA. And the application would be very quick in a limited time.

The RD technique ${ }^{[8]}$ was initially a method for gene separating and differential display. As ds cDNAs were digested by the restricted enzyme and ligated with adaptors on both ends, the samples could be labeled by a fluorescent universal primer through PCR amplifications. Sample fragments labeled by this method had a fluorescent molecule on both ends, which determined the linear relation between the intensity of fluorescence and the quantity of PCR products. This would be helpful in reflecting the original status of the samples. Moreover, the hybridization condition would be easier to control and the signal to noise ratio is lower because fragments prepared by this method were more uniform in length. In addition, these methods could decrease the consumption of fluorescent dyes compared with the incorporation labeling by fluorescent-dNTP, which could reduce the cost of microarray experiments and be advantageous to clinical applications.

\section{References}

1. Peiris, J., Lai, S., Poon, L. et al., Coronavirus as a possible cause of severe acute respiratory syndrome, Lancet, 2003, 361(9366): $1319-1325$.

2. Ksiazek, T. G., Erdman, D., Goldsmith, C. S. et al., A novel coronavirus associated with severe acute respiratory syndrome, $\mathrm{N}$. Engl. J. Med., 2003, 348(20): 1953-1966.

3. Rota, P. A., Oberste, M. S., Monroe, S. S. et al., Characterization of a novel coronavirus associated with severe acute respiratory syndrome, Science, 2003, 300(5624): 1394-1399.

4. Marra, M. A., Jones, S. J., Astell, C. R. et al., The genome sequence of the SARS-associated coronavirus, Science, 2003, 300(5624): $1399-1404$.

5. Qin, E. D., Zhu, Q. Y., Yu, M. et al., A complete sequence and comparative analysis of a SARS-associated virus (Isolate BJ01), Chinese Science Bulletin, 2003, 48(10): 941-948.

6. Wang, Y., Ma, W. L., Song, Y. B. et al., Gene sequence analysis of SARS-associated coronavirus by nested RT-PCR, Di Yi Jun Yi Da Xue Xue Bao (in Chinese), 2003, 23(5): 421-423.

7. Shi, R., Ma, W. L., Song, Y. B. et al., Two restriction fluorescence labeling methods for enhancing the signal-to-noise ratio of cDNA microarray hybridization, Di Yi Jun Yi Da Xue Xue Bao (in Chinese), 2003, 23(2): 124-126.

8. Zhang, B., Ma, W. L., Wu, Q. H., Construction of a cDNA fragment library from SH-SY5Y cells using restriction display PCR, Br. J. Biomed. Sci., 2002, 59(1): 35-37.

(Received May 13, 2003; accepted June 11, 2003) 